\title{
Ambient Vibration Test of a 18-Story Reinforced Concrete Building
}

\author{
Jun $\mathrm{Ma}^{\mathrm{a}}$, Jie Ge \\ China Construction Eighth Engineering Division Co., Ltd., Engineering Research Institute, 200122 Shanghai, China
}

\begin{abstract}
Ambient vibration analysis of a 18-story reinforced concrete building was carried out to investigate its dynamic properties. Ambient vibration test was conducted on the building, and modal parameters including natural frequencies and damping ratios were identified using the Frequency domain decomposition (FDD) method and the Enhanced frequency domain decomposition (EFDD) method. The results demonstrate that ambient vibration test is a feasible and efficient technique to excite structures, and that FDD and EFDD methods are reliable and efficient techniques for ambient vibration data.
\end{abstract}

\section{Introduction}

Ambient vibration test is a key technology in estimating dynamic properties of civil engineering structures. Modal parameters including natural frequencies, damping ratios, and mode shapes are extracted from ambient vibration responses of a structure, and can be used for a number of practical applications, such as structural vibration control, structural modal updaing, and damage assessment. Ambient vibration test requires almost no equipment to excite structures, and no damage will be caused during test procedures, thus it is available for almost all kinds of civil engineering structures.

This paper investigates the dynamic properties of a newly designed and constructed 18-story reinforced concrete building by ambient modal analysis. Ambient vibration test was conducted, and Frequency domain decomposition (FDD) method and Enhanced frequency domain decomposition (EFDD) method were employed to evaluate modal parameters. The procedure of applying FDD and EFDD methods were presented. The purpose of this paper is to assess the feasibility of ambient vibration test technique for civil engineering structures and to evaluate the reliability and efficiency of FDD and EFDD methods for ambient vibration data.

\section{Ambient Vibration Test}

The test building is a 18-story residence building located in Shanghai. It has one story beneath ground level and 18 stories above, and extends from $5.3 \mathrm{~m}$ underground to $53.1 \mathrm{~m}$ above basement level, as show in Figure 1. The building is supported by reinforced concrete shear walls at the core and around the perimeter, as shown in Figure 2 . The shear walls are $200 \mathrm{~mm}$ thick and provide majority of lateral strength and stiffness for the building. Concrete slabs with the thickness of $110 \mathrm{~mm}$ are adopted throughout the entire structure. Autoclaved aerated concrete (AAC) blocks are used for exterior walls and interior walls. The plan of a standard story is $22.2 \mathrm{~m}$ long by $11.4 \mathrm{~m}$ wide, and the story height is $2.95 \mathrm{~m}$.

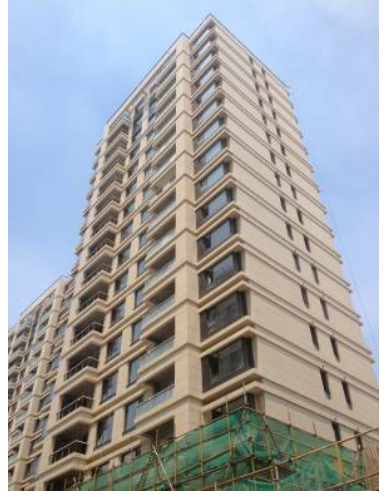

Figure 1. Test building

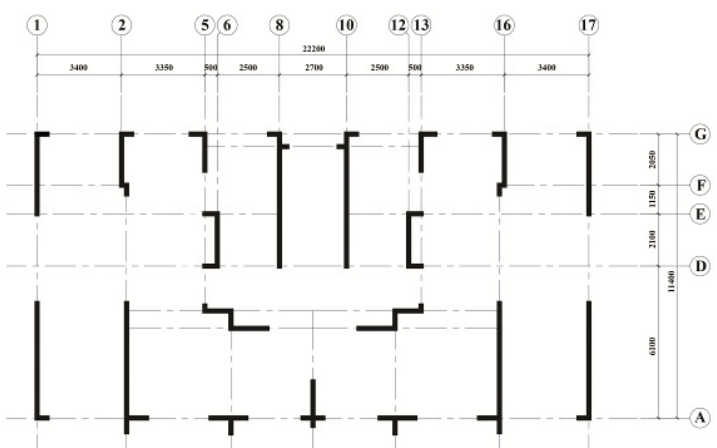

Figure 2. Plan view of a standard story

Ambient vibration test on this building was conducted on July, 2017. 3 servo-type accelerometers with a 16 channel data acquisition system were placed on the top story to measure acceleration responses of the building induced by wind, traffic and operational activities. Two accelerometers were set horizontally, one along the length direction and the other along the width direction, and the remaining accelerometer was set vertically. The accelerometers were set to a sampling

\footnotetext{
* Corresponding author: ${ }^{a}$ mjbppc_academic@email.com
} 
rate of $100 \mathrm{~Hz}$, and the duration of each record was 3,600 seconds, thus a total length of 360,000 data points were recorded for each channel. The two horizontal accelerometers were shown in Figure 3, and the data acquisition system was shown in Figure 4.

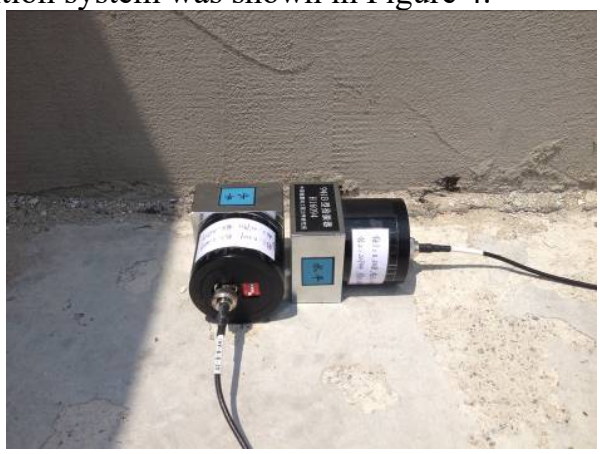

Figure 3. Horizontal accelerometers

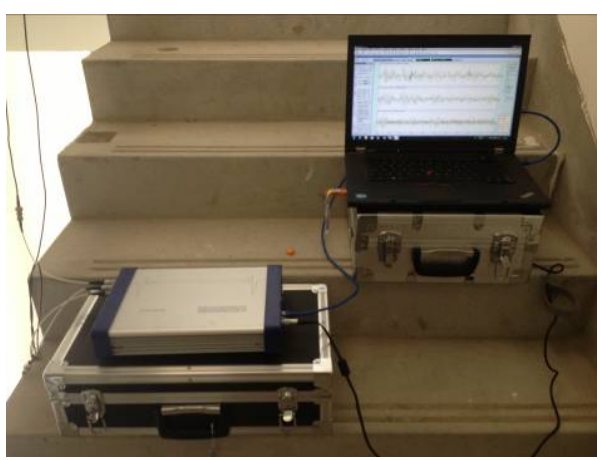

Figure 4. Data acquisition system

\section{Ambient Vibration Analysis}

Frequency domain decomposition method and Enhanced frequency domain decomposition method were adopted to extract modal information from ambient vibration data. Frequency domain decomposition method is an extension of the Peak picking (PP) method by taking advantage of Singular value decomposition (SVD) [1-5]. In FDD method, instead of using Power spectral density (PSD) directly, the output response PSD matrix is decomposed at discrete frequencies by taking SVD of the matrix,

$$
G_{\mathrm{yy}}\left(j \omega_{\mathrm{i}}\right)=U_{\mathrm{i}} S_{\mathrm{i}} U_{\mathrm{i}}^{\mathrm{H}}
$$

where $G_{\mathrm{yy}}\left(j \omega_{\mathrm{i}}\right)$ is the output matrix, $U_{\mathrm{i}}=\left[u_{\mathrm{i} 1}, u_{\mathrm{i} 2}, \ldots, u_{\mathrm{im}}\right]$ is a unitary matrix holding the singular vectors $u_{\mathrm{ij}}$, and $S_{\mathrm{i}}$ is a diagonal matrix holding the scalar singular values $s_{\mathrm{ij}}$.

The output response PSD matrix is decomposed into a set of auto-PSD functions, each function corresponds to a Single degree of freedom (SDOF) system. Natural frequencies and mode shapes can be extracted from the matrix $S_{\mathrm{i}}$ and the matrix $U_{\mathrm{i}}$. Peaks of the first singular values $s_{\mathrm{i} 1}$ are equal to natural frequencies of the structure, and singular vectors corresponding to the peaks of the first singular values are approximations of mode shape vectors. These approximations become exact in the case where the input force is white noise, the structure is lightly damped, and mode shapes of those closely spaced modes are geometrically orthogonal. If these assumptions are not satisfied, the decomposition of PSD matrix to a set of PSD functions is still expected to be more accurate than those of the classical approaches. The advantage of FDD is that it allows identification of coupled modes that are easy to lose as they appear on individual frequency spectrum. In the case of coupled modes, there will be as many dominating singular values as the coupled modes.

Enhanced frequency domain decomposition method is an improvement of the FDD method. While FDD method gives natural frequencies and mode shapes of structures, EFDD method gives an improved estimation of natural frequencies, mode shapes, and damping ratios [6-8]. In EFDD method, the PSD functions identified around peaks of the first singular values are taken back to the time domain using the Inverse discrete Fourier transform (IDFT), then natural frequencies can be obtained by the crossing times, and damping ratios can be estimated using logarithmic decrement of the SDOF normalized auto correlation functions, which are results of the IDFT.

The SDOF PSD functions are identified around peaks of the first singular values using the Modal assurance criterion (MAC). The first singular vectors around those peaks are compared with the first singular vectors at corresponding peaks, and the MAC values are calculated, here the first singular vectors at the peaks are used as reference vectors in MAC. If MAC values are higher than a user-specified threshold, which is generally set from 0.80 to 1.00 , the corresponding singular values are included in the SDOF PSD function.

The MAC equation is written as,

$$
M A C=\frac{\left|\psi^{T} \phi\right|}{\left(\psi^{T} \psi\right)\left(\phi^{T} \phi\right)}
$$

where $\Psi$ is the first singular vectors around peaks of the first singular values, $\Phi$ is the first singular vectors at corresponding peaks.

\section{Results of Ambient Vibration Analysis}

Modal parameters of the building were identified using the FDD and EFDD method. The measured response acceleration data along the width direction and along the length direction, as well as the associated PSD functions, are shown in Figure 5 and Figure 6, respectively. The response acceleration data is quite small, most of the measured data is in the -10 to 10 millimeter per second squared range. The PSD functions show distributions of modes of these two signals, it can be observed that modes are scatteredly distributed.

Singular values of the output response PSD matrix are obtained using the FDD method and are shown in the frequency range from $0 \mathrm{~Hz}$ to $10 \mathrm{~Hz}$ in Figure 7. The close modes are clearly indicated in the plot. The first singular values are used to obtain natural frequencies by peak picking, and the first six natural frequencies are indicated in the figure and are listed in Table 1. As shown in the figure, the first mode and the second mode are two coupled modes, the identified first-order frequency is $1.07 \mathrm{~Hz}$ and the second-order frequency is 
$1.27 \mathrm{~Hz}$, the fourth mode and the fifth mode are also closely spaced, the fourth-order frequency is $4.98 \mathrm{~Hz}$ and the fifth-order frequency is $5.47 \mathrm{~Hz}$. Coupled modes are identified with the application of FDD method.

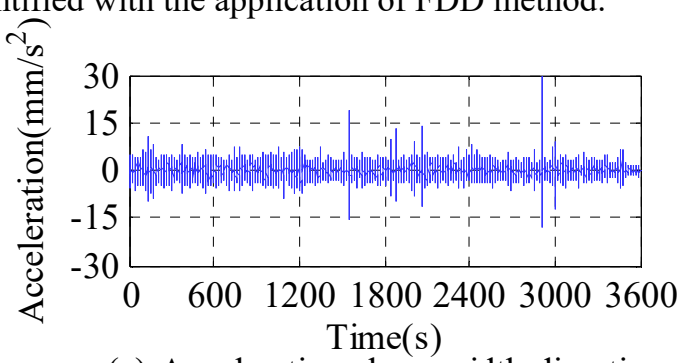

(a) Acceleration along width direction

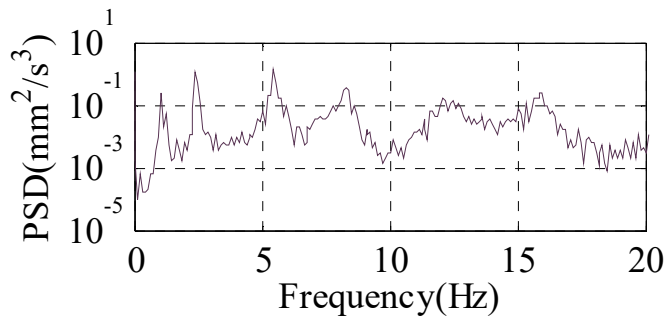

(b) Power spectral density

Figure 5. Acceleration record along width direction

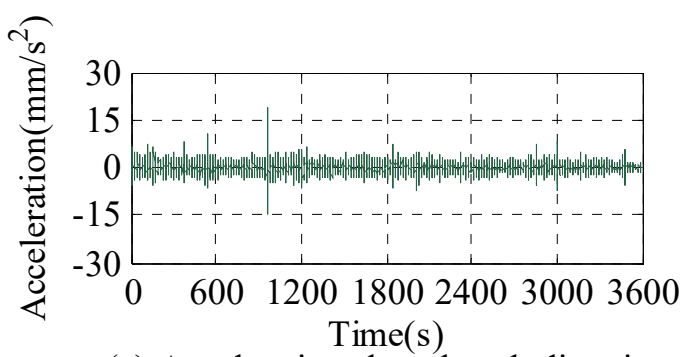

(a) Acceleration along length direction

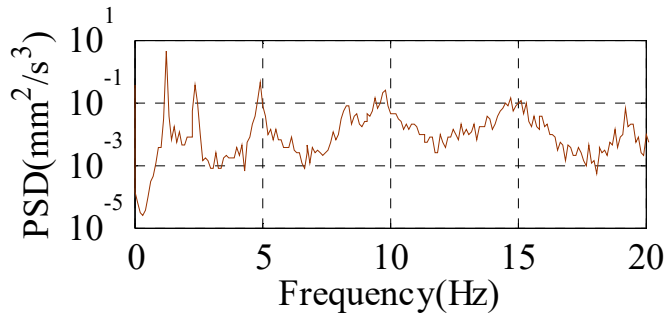

(b) Power spectral density

Figure 6. Acceleration record along length direction

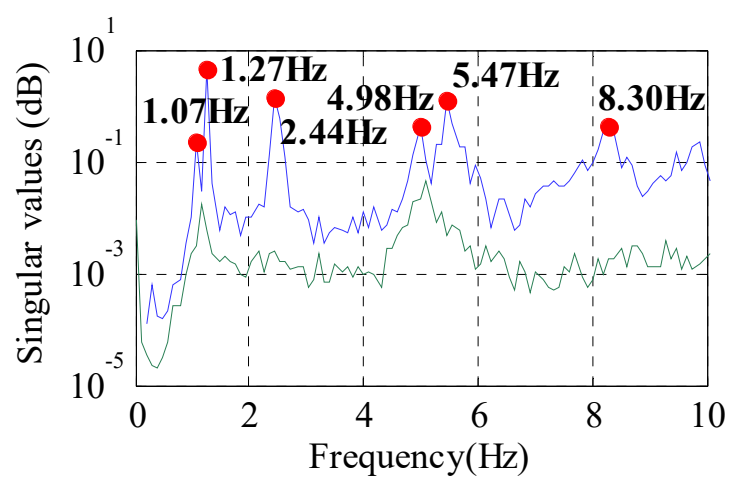

Figure 7. Singular values of the output response PSD matrix

EFDD method is then adopted to estimate damping ratios, the first six damping ratios are estimated and are listed in Table 1. The procedure of estimating damping ratio of the first mode is shown in Figures 8 to 10 .
The singular values corresponding to the first mode are obtained using the MAC technique, a reasonable part of the PSD functions is identified, as shown in Figure 8. A threshold of 0.80 is set to determine those singular values belonging to the first mode. The estimated SDOF is within the scope of $0.49 \mathrm{~Hz}$ to $1.95 \mathrm{~Hz}$.

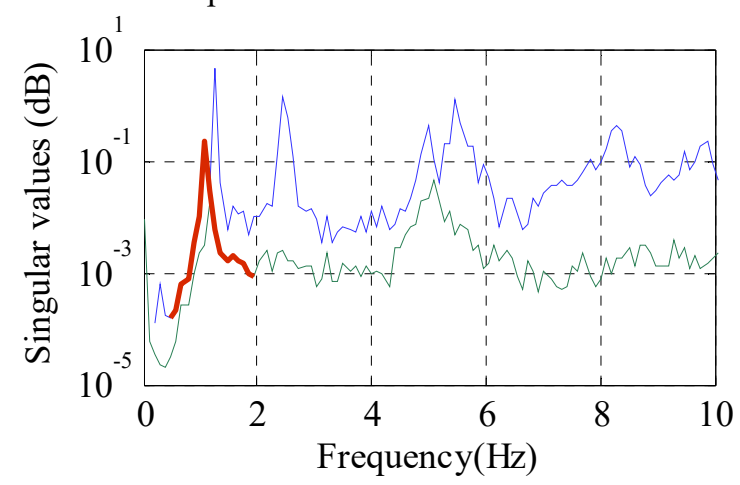

Figure 8. Identification of the first SDOF system

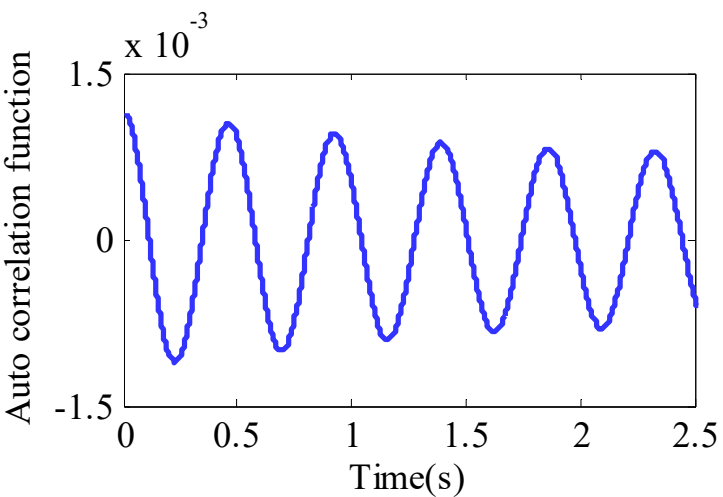

Figure 9. Normalized autocorrelation function of the first mode

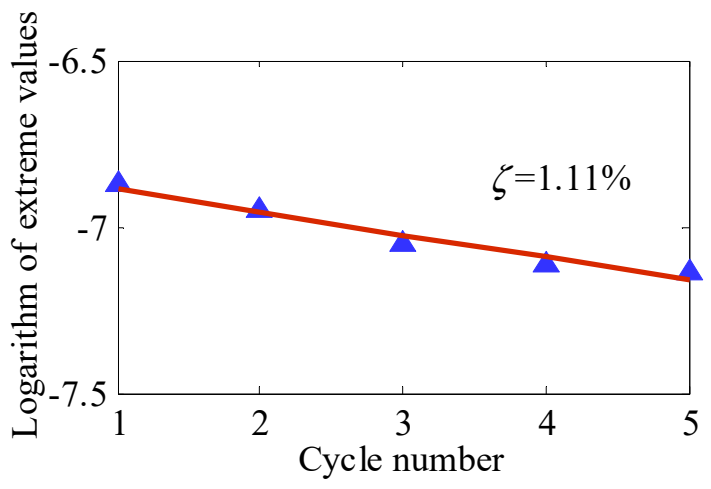

Figure 10. Estimation of damping ratio of the first mode

The normalized auto correlation function is obtained by performing an IDFT on the above SDOF functions. Figure 9 shows the free decay function in the time domain. The damping ratio is then estimated by the logarithmic decrement technique, as shown in Figure 10. The estimation is performed by applying a linear fit between the positive peaks extracted from the free decay function and the associated integer number of successive, positive peaks. The damping ratio is estimated to be $1.11 \%$. 
Table 1. Natural frequencies and damping ratios of the building.

\begin{tabular}{|c|c|c|}
\hline Mode & Natural frequency (Hz) & Damping ratio (\%) \\
\hline $1^{\text {st }}$ & 1.07 & 1.11 \\
\hline $2^{\text {nd }}$ & 1.27 & 0.81 \\
\hline $3^{\text {rd }}$ & 2.44 & 1.36 \\
\hline $4^{\text {th }}$ & 4.98 & 1.35 \\
\hline $5^{\text {th }}$ & 5.47 & 1.31 \\
\hline $6^{\text {th }}$ & 8.30 & 2.29 \\
\hline
\end{tabular}

\section{Conclusions}

In this paper ambient vibration test was conducted on a 18-story reinforced concrete building, and dynamic properties including natural frequencies and damping ratios were identified by the FDD and EFDD method. The procedure of applying FDD and EFDD methods to evaluate modal parameters was presented, and the estimated results were exhibited.

Based on the identified results, it can be concluded that ambient vibration test is an efficient method to excite structures, and that FDD and EFDD method are efficient techniques to extract modal information from ambient vibration data and to give reliable estimations of modal parameters. FDD method can be used to extract modal information from ambient vibration data, and it has an advantage in identifying close or repeated modes. EFDD method extends the application of FDD method in that it leads to an estimation of both frequency and damping, and the damping estimation can be controlled by adjusting the MAC value. As ambient vibration tests are more often used in field measurements of civil engineering structures, it is of utmost importance to assess its feasibility and to evaluate the reliability of mode identification methods.

\section{References}

1. R. Brincker, L.M. Zhang, P. Andersen, Smart Mater. Struct. 10, 3(2001).

2. M.Q. Feng, D.K. Kim, J.H. Yi, Y.B. Chen, J. Eng. Mech. 130, 5(2004).

3. R. Brincker, P. Andersen, N-J. Jacobsen, 25th International Modal Analysis Conference (Society for Experimental Mechanics, Orlando, 2007).

4. M. Mieloszyk, S. Opoka, W. Ostachowicz, 11th International Conference on Damage Assessment of Structures (IOP Publishing, Ghent, 2015).

5. R. Tarinejad, M. Damadipour, J. Sound Vib. 333, 3(2014).
6. R. Brincker, C.E. Ventura, P. Andersen, 19th International Modal Analysis Conference (Society for Experimental Mechanics, Kissimmee, 2001).

7. N-J. Jacobsen, P. Andersen, R. Brincker, International Conference on Noise and Vibration Engineering (KU Leuven, Leuven, 2006).

8. B. Sevim, A. Bayraktar, A.C. Altunişik, S. Adanur, M. Akköse, J. Test. Eval. 38, 5(2010). 